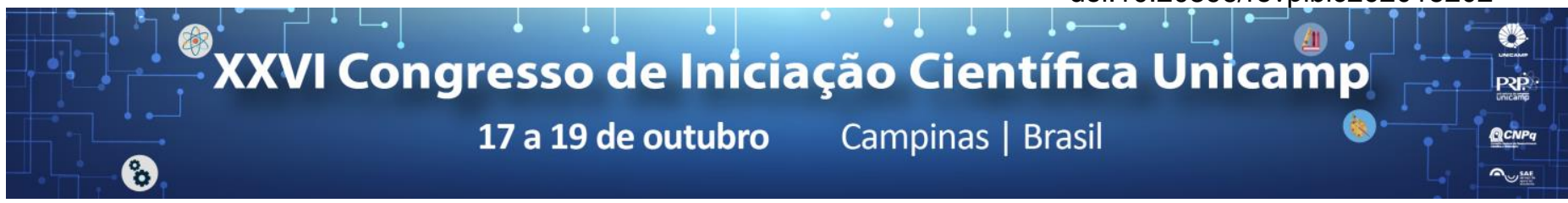

\title{
Avaliação objetiva da qualidade de imagem em sistemas de placa de fósforo fotoestimuláveis
}

\section{Beatriz P. Negri, Amanda P. Candemil, Francisco Haiter-Neto, Deborah Q. de Freitas, Matheus Lima Oliveira.}

\section{Resumo}

O objetivo deste estudo foi de avaliar a qualidade da imagem de forma objetiva em sistemas radiográficos digitais de placa de fósforo fotoestimulável (PSP). Um fantoma de imagem foi radiografado com o aparelho de raios $X$ Focus ${ }^{\circledR}$ ajustado em $70 \mathrm{kVp}, 7 \mathrm{~mA}$, tempos de exposição de $0,32,0,63,0,80 \mathrm{~s}$ e modos de resolução "low" (RL) e "high" (RH). Dez repetidas aquisições foram realizadas em três sistemas de PSP (Express, Digora, VistaScan) para cada protocolo. Todas as imagens foram avaliadas no software ImageJ. Por meio do valor médio de pixel foram calculados o ruído, uniformidade e relação sinal-ruído (SNR). Os dados foram submetidos a análise de variância e teste de Tukey com nível de significância de $5 \%$. Os valores de ruído no sistema vistascan foram significativamente maiores e no sistema express significativamente menores. Os valores de uniformidade no sistema digora foram significativamente maiores e no sistema express significativamente menores. Os valores de SNR no sistema vistascan foram significativamente menores e no sistema express foram significativamente maiores (tabelas 1, 2 e 3). Concluiu-se que o sistema Express de PSP produziu imagens com menor ruído e maior homogeneidade de valores de pixel.

\section{Palavras-chave:}

Radiografia dentária digital, sensibilidade de contraste, técnicas in vitro.

\section{Introdução}

A radiografia convencional vem sendo substituída pela radiografia digital na odontologia. ${ }^{1,2}$ Pesquisas recentes evidenciam a importância da avaliação da qualidade de imagem dos receptores digitais para que se mantenha uma alta acurácia no diagnóstico. .,4 $^{0}$ objetivo deste estudo foi de avaliar a qualidade da imagem de forma objetiva em sistemas radiográficos digitais de placa de fósforo fotoestimulável (PSP).

\section{Resultados e Discussão}

Os valores de ruído no sistema express foram significativamente maiores com 0,32 s na $R L$ e com 0,32 e $0,8 \mathrm{~s}$ na $\mathrm{RH}$. No sistema digora, com $\mathrm{RH}$, os valores foram significativamente maiores com 0,63 e 0,8 s. Em relação a resolução no sistema express, os valores foram significativamente maiores na $\mathrm{RH}$ com $0,8 \mathrm{~s}$ (tab. 1). Os valores de uniformidade no sistema digora com $R L$ foram significativamente maiores com $0,8 \mathrm{~s}$. Nos sistemas vistascan e digora, com $\mathrm{RH}$, os valores foram significativamente maiores com $0,63 \mathrm{~s}$. Em relação a resolução, no sistema vistascan, os valores foram significativamente maiores na $R L$ com 0,32 e 0,8 s e na $R H$ com 0,63 s (tab. 2). Os valores de SNR no sistema express foram significativamente maiores com 0,63 e 0,8 s na $R L$ e 0,63 s na $\mathrm{RH}$. Em relação a resolução, os valores no sistema express foram significativamente maiores em $R L$ (tab. 3).

\begin{tabular}{cccc}
\multicolumn{4}{l}{ Tabela 1 - Valores de ruído } \\
\hline Sistema & Tempo & Low & High \\
\hline \multirow{2}{*}{ Express $^{\mathrm{C}}$} & 0,32 & $10,60^{\mathrm{Aa}}$ & $12,54^{\mathrm{Aa}}$ \\
\cline { 2 - 4 } & 0,63 & $7,38^{\mathrm{Ba}}$ & $8,92^{\mathrm{Ba}}$ \\
\cline { 2 - 4 } & 0,8 & $8,20^{\mathrm{AB}}$ & $12,34^{\mathrm{Aa}}$ \\
\hline \multirow{2}{*}{ Digora $^{\mathrm{B}}$} & 0,32 & $13,97^{\mathrm{Aa}}$ & $13,51^{\mathrm{Ba}}$ \\
\cline { 2 - 4 } & 0,63 & $15,03^{\mathrm{Aa}}$ & $16,52^{\mathrm{Aa}}$ \\
\cline { 2 - 4 } & 0,8 & $15,45^{\mathrm{Aa}}$ & $15,56^{\mathrm{Aa}}$ \\
\hline \multirow{2}{*}{ Vista $^{\mathrm{A}}$} & 0,32 & $31,88^{\mathrm{Aa}}$ & $31,70^{\mathrm{Aa}}$ \\
\cline { 2 - 4 } & 0,63 & $31,80^{\mathrm{Aa}}$ & $31,09^{\mathrm{Aa}}$ \\
\cline { 2 - 4 } & 0,8 & $10,60^{\mathrm{Aa}}$ & $12,54^{\mathrm{Aa}}$ \\
\hline
\end{tabular}

Tabela 2- Valores de uniformidade

\begin{tabular}{cccc}
\hline Sistema & Tempo & Low & High \\
\hline \multirow{3}{*}{ Express $^{\mathrm{C}}$} & 0,32 & $5,43^{\mathrm{Aa}}$ & $7,72^{\mathrm{Aa}}$ \\
\cline { 2 - 4 } & 0,63 & $5,30^{\mathrm{Aa}}$ & $5,00^{\mathrm{Aa}}$ \\
\cline { 2 - 4 } & 0,8 & $5,35^{\mathrm{Aa}}$ & $10,33^{\mathrm{Aa}}$ \\
\hline \multirow{3}{*}{ Digora $^{\mathrm{A}}$} & 0,32 & $14,58^{\mathrm{Ba}}$ & $12,52^{\mathrm{Ca}}$ \\
\cline { 2 - 4 } & 0,63 & $17,42^{\mathrm{ABa}}$ & $20,04^{\mathrm{Aa}}$ \\
\cline { 2 - 4 } & 0,8 & $18,72^{\mathrm{Aa}}$ & $16,70^{\mathrm{Ba}}$ \\
\hline \multirow{3}{*}{ Vista $^{\mathrm{B}}$} & 0,32 & $7,15^{\mathrm{Aa}}$ & $3,57^{\mathrm{Cb}}$ \\
\cline { 2 - 4 } & 0,63 & $7,48^{\mathrm{Ab}}$ & $22,08^{\mathrm{Aa}}$ \\
\cline { 2 - 4 } & 0,8 & $7,58^{\mathrm{Aa}}$ & $5,96^{\mathrm{Bb}}$ \\
\hline
\end{tabular}

Tabela 3- Valores de SNR

\begin{tabular}{cccc}
\hline Sistema & Tempo & Low & High \\
\hline \multirow{2}{*}{ Express $^{\mathrm{A}}$} & 0,32 & $9,49^{\mathrm{Ba}}$ & $8,14^{\mathrm{Bb}}$ \\
\cline { 2 - 4 } & 0,63 & $13,91^{\mathrm{Aa}}$ & $11,38^{\mathrm{Ab}}$ \\
\cline { 2 - 4 } & 0,8 & $13,27^{\mathrm{Aa}}$ & $9,03^{\mathrm{Bb}}$ \\
\hline \multirow{2}{*}{ Digora $^{\mathrm{B}}$} & 0,32 & $7,27^{\mathrm{Aa}}$ & $7,47^{\mathrm{Aa}}$ \\
\cline { 2 - 4 } & 0,63 & $6,85^{\mathrm{Aa}}$ & $6,24^{\mathrm{Aa}}$ \\
\hline \multirow{3}{*}{ Vista $^{\mathrm{C}}$} & 0,8 & $6,61^{\mathrm{Aa}}$ & $6,48^{\mathrm{Aa}}$ \\
\cline { 2 - 4 } & 0,32 & $3,14^{\mathrm{Aa}}$ & $3,16^{\mathrm{Aa}}$ \\
\hline & 0,63 & $3,14^{\mathrm{Aa}}$ & $3,21^{\mathrm{Aa}}$ \\
\hline
\end{tabular}

\section{Conclusões}

Concluiu-se que o sistema Express de PSP produziu imagens com menor ruído e maior homogeneidade de valores de pixel.

\section{Agradecimentos}

Conselho Nacional de Desenvolvimento Científico e Tecnológico (CNPq).

1 Udupa, H; Mah, P; Dove, SB; McDavid, WD. Evaluation of image quality parameters of representative intraoral digital radiographic systems. Oral Surgery Oral Medicine Oral Pathology Oral Radiology 2013; 116: 774-783.

${ }^{2}$ Haiter-Neto, F; Melo, DP. Radiografia Digital. Revista da ABRO 2010; 11:517.

${ }^{3}$ Olsson, L; Nilsson, M; Svenson, B; Hellén-Halme, K. The effetc of anatomical noise on perception of low contrast in intra-oral radiographs: an in vitro study. Dentomaxillofacial Radiology 2016; 45:20150402.

${ }^{4}$ Hellén-Halme, K; Johansson, C; Nilsson, M. Comparison of the performance of intraoral Xray sensors using objective image quality assessment. Oral Surgery Oral Medicine Oral Pathology Oral Radiology 2016; 121:129-137. 